Article

\title{
Design and Dynamic Simulation Analysis of a Wheel-Track Composite Chassis Based on RecurDyn
}

\author{
Yaowei Li ${ }^{1}$, Liguo Zang ${ }^{1, *}$, Tuo Shi ${ }^{1}$, Tian $\mathrm{Lv}^{1}$ and Fen Lin ${ }^{2}$ \\ 1 School of Automobile and Rail Transportation, Nanjing Institute of Technology, Nanjing 211167, China; \\ liyaowei051129@163.com (Y.L.); shituo_zxy@163.com (T.S.); lit0202@163.com (T.L.) \\ 2 College of Energy and Power Engineering, Nanjing University of Aeronautics and Astronautics, \\ Nanjing 210016, China; flin@nuaa.edu.cn \\ * Correspondence: zangliguo@njit.edu.cn
}

check for updates

Citation: Li, Y.; Zang, L.; Shi, T.; Lv, T.; Lin, F. Design and Dynamic Simulation Analysis of a WheelTrack Composite Chassis Based on RecurDyn. World Electr. Veh. J. 2022, 13, 12. https://doi.org/10.3390/ wevj13010012

Academic Editor: Joeri Van Mierlo

Received: 21 November 2021

Accepted: 22 December 2021

Published: 31 December 2021

Publisher's Note: MDPI stays neutral with regard to jurisdictional claims in published maps and institutional affiliations.

Copyright: (C) 2021 by the authors. Licensee MDPI, Basel, Switzerland. This article is an open access article distributed under the terms and conditions of the Creative Commons Attribution (CC BY) license (https:// creativecommons.org/licenses/by/ $4.0 /)$.

\begin{abstract}
The traditional chassis has the problems of low trafficability and poor stability under complex and changeable unstructured conditions. Thus, a wheel rail composite chassis is proposed. The chassis had a tracked travel mechanism at the front wheels and a wheeled travel mechanism at the rear wheels. This study presents the design, theoretical analysis and dynamic simulation analysis of the chassis. The maximum values of the passability of the wheel-track composite chassis that can be passed were calculated according to the relevant parameters. Furthermore, the chassis was modeled and simulated using RecurDyn to verify whether the values were reasonable. According to different values of the terrain, slope, vertical obstacle height and trench width, the change regularity of the track tension and driving torque of the chassis were obtained. The chassis is designed to improve the vehicle's ability to operate under complex and diverse unstructured conditions.
\end{abstract}

Keywords: wheel-track composite chassis; dynamic simulation; RecurDyn; climbing performance; obstacle crossing performance

\section{Introduction}

A single-wheeled travel mechanism is widely used in the traditional motor vehicle chassis system. Its theoretical technology is more mature, and it has simple control and flexible steering with high mobility, but the obstacle climbing performance is relatively poor. A single-tracked travel mechanism has a large ground contact area, and its ground pressure is smaller so that it is not easy to slip and sink on slippery and soft ground. A single-tracked travel mechanism has better obstacle climbing performance, but compared with that of single wheel, the mobility is poor and the steering is heavy [1,2].

There are a vast territory and a wide variety of terrains in China. Therefore, the traditional chassis system is difficult to pass through terrain such as swamp, hills and sandy terrain [3]. In order to solve this problem, a wheel-track composite chassis system was designed, which also has two advantages of high mobility of wheel chassis and high passage of track chassis. It not only can improve the environmental adaptability of vehicles but also can expand the work scope of vehicles, which can make up for the defects of the traditional chassis system. In addition, it is widely used in post-disaster rescue, agricultural production and other occasions [4].

Since the 20th century, many foreign universities, enterprises and scholars have carried out in-depth research on the wheel-track composite chassis and have achieved corresponding results [3]. Wang et al. [5] designed a wheel-track combination floor climbing wheelchair to cross stairs of about 33 degrees and rugged roads with a vertical height of $245 \mathrm{~mm}$ and a trench width of $430 \mathrm{~mm}$. The mechanical system of the wheelchair mainly includes a wheel-tracked walking mechanism, attitude transformation and power switching mechanism, compound power box, seat, seat balance adjustment mechanism, etc. A new design of a composite mobile chassis is proposed by Li et al. [6], and the variant wheel 
in the chassis system has a telescopic device that can turn the reconfigurable track into a triangle to increase the contact area with the ground and improve its passing performance. Bian et al. [7] studied a reconnaissance search robot based on deformation tracks, which changes the angle between the tracks and the ground by changing the relative position of the wheels attached to the tracks, thus changing the shape of the tracks. A team of students and mentors at the University of Ljubljana [8] have developed a concept for a composite wheelchair, which is designed with independent steering mechanisms for each wheel and wheel-driven tracks for better stair climbing, allowing efficient mobility and maneuverability in both indoor and outdoor environments. Carnegie Mellon University's National Robotics Engineering Center [9] has researched an adaptive deformable wheel that can be converted from an ordinary round wheel to a triangular-shaped tracked wheel in a very short period of time through a special telescoping mechanism. The vehicle does not need to stop during the conversion process, and the adaptability to different terrains can be enhanced in a timely manner, thus greatly improving the efficiency of the vehicle.

The wheel-track composite robot researched at the Gyeongbuk Institute of Science and Technology in Daegu, Korea [10], is used to perform surveillance, reconnaissance and search and rescue tasks in hazardous environments. It consists of a common wheeled structure and a variable-tracked structure with good performance for fast navigation on flat ground and overcoming stairs or obstacles. The AZIMUT robot from the University of Sherbrooke in Canada [11] can choose the movement mode according to the environment, and the robot overcomes obstacles by changing the height of its legs. When encountering rough and muddy roads, the robot can switch to track mode to improve passability. When the robot travels on a flat surface, it can choose the wheeled mode to improve its flexibility and agility. Kim and some others of Jeonbuk National University in Korea proposed a new rescue robot with a changeable track structure [12], which used a new travel mechanism to accomplish various shape changes of the track mechanism to achieve gains in steering and rotation as well as the ability to go over stairs.

In this paper, the research object of wheel-track composite chassis was taken to conduct theoretical analysis on its passing performance, and a three-dimensional model of the chassis for simulation analysis was established. Hence, the reasonableness of the maximum slope value, the highest vertical obstacle and the widest trench depth value that can be passed was verified; additionally, the change law of track tension and driving torque were summarized during the driving process of the chassis.

\section{System Composition}

The travel mechanism of the wheel-track composite chassis consists of track and a wheel, respectively. The tracked travel mechanism is composed of a driving wheel, a guide wheel and other components, and the wheeled travel mechanism is composed of tires and rims, etc. The composition of the travel mechanism is introduced in detail in this section.

\subsection{Overall Structure Design and Working Principle}

In this section, the structural components of the tracked travel mechanism and the wheeled travel mechanism are introduced separately.

\subsubsection{Design of Tracked Travel Mechanism}

The front wheel of the chassis is designed as a triangular tracked wheel. As shown in Figure 1, it includes a driving wheel, a guide wheel, a tensioning wheel and three branch weight wheels. When the vehicle is running, the engine transmits a certain torque to the driving wheel, and the driving wheel engages with the tracks to drive the tracks, so that the tracked wheels overcome a series of resistances to move forward [13]. The steering wheel has a guiding effect on the track, which can avoid the track from running off and crossing the track in the process of driving and can also increase the contact area of the track and reduce the grounding ratio pressure [14]. The tensioning wheel can adjust the tension of the track through its own swing, reducing the wear caused by the track relaxation or 
tightening, and thus can achieve better passing through the complex terrain $[15,16]$. The weight of the whole vehicle is carried by supporting wheels [17], which restrain the lateral movement of the tracks and withstand the ground impact [18].

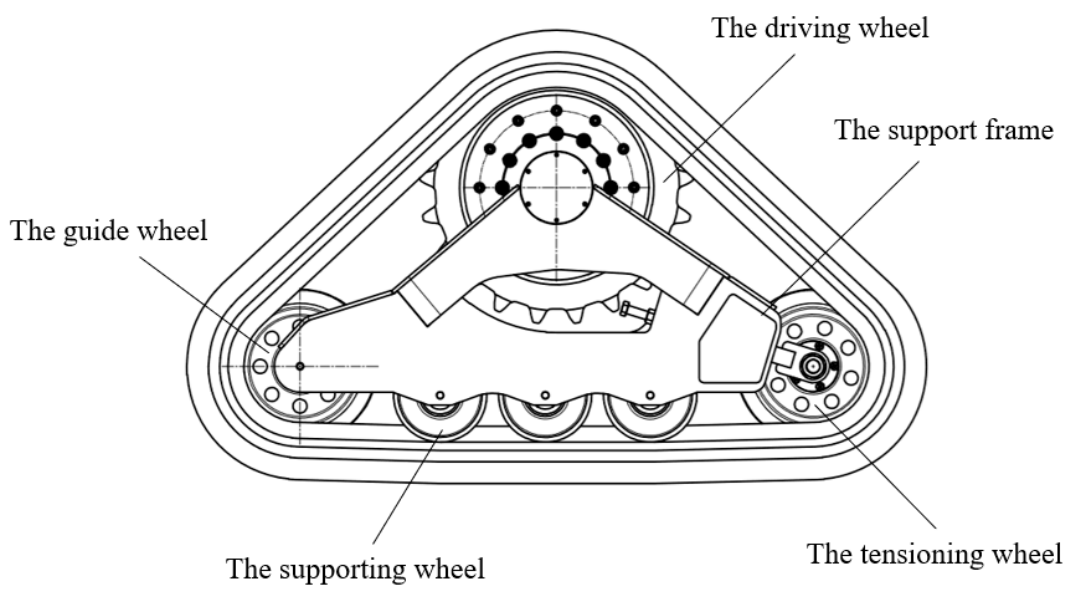

Figure 1. Structural diagram of the triangular tracked wheel.

\subsubsection{Design of Wheeled Travel Mechanism}

The rear wheel of the chassis is designed as a wheeled travel mechanism composed of glue complex and curtain line, etc. The wheeled travel mechanism is widely used in modern vehicles due to its good maneuverability. During the driving process, the circular tire needs to bear the rear load of the vehicle, and the adhesion between the wheels and the ground has a deceleration and absorption effect on the vibration and impact of the vehicle, thus preventing the vehicle parts from violent vibration and reducing the service life. In addition, the tire also has traction and braking functions to transmit the power of the engine or brake to the road, while it can also steer and stabilize the vehicle to turn in the desired direction or remain straight [19]. As shown in Figure 2, it includes tire, rim and some other components.

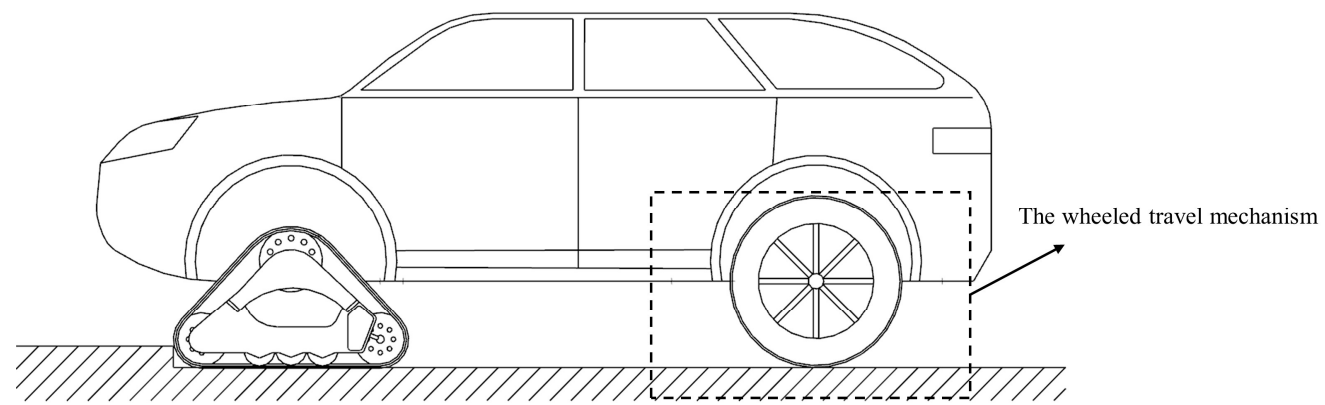

Figure 2. Structural diagram of the wheel.

\subsection{Design of the Triangular Tracked Wheel}

The triangular tracked wheel includes a driving wheel, a guide wheel, three supporting wheels, a tensioning wheel, a support frame and rubber tracks. The driving wheel is installed above the support frame, the guide wheel and the tensioning wheel are installed on the left and right edges of the support frame and three supporting wheels are installed at the bottom of the support frame. The radius of the driving wheel is an important parameter of the driving wheel, which is directly related to the driving torque and driving speed of the tracked vehicle. Additionally, the driving radius is proportional to the driving torque; when the driving wheel speed is constant, the radius of the driving wheel is proportional to the driving speed [20]. The track parameters are shown in Table 1. 
Table 1. Parameters table of tracked travel mechanism.

\begin{tabular}{ccc}
\hline Track Parameters/mm & Abbreviation & Value \\
\hline Track pitch & $\mathrm{p}$ & 150 \\
Track width & $\mathrm{b}$ & 400 \\
The axial distance between the & $\mathrm{l}_{1}$ & 2300 \\
driving wheel and the guide wheel & $\mathrm{B}$ & 1600 \\
Track height & $\mathrm{H}$ & 970 \\
Total width of the chassis & $\mathrm{C}$ & 2000 \\
Track ground length & $\mathrm{L}_{0}$ & 1100 \\
Spacing between rear supporting & $\mathrm{C}_{2}$ & 350 \\
wheel and drive wheel & & \\
Spacing between front supporting & $\mathrm{C}_{1}$ & 300 \\
wheel and drive wheel & & 450 \\
Spacing between two supporting & $\mathrm{l}_{0}$ & \\
wheels at both ends & & 225 \\
Spacing between two adjacent & $\mathrm{t}_{1}$ & 331 \\
supporting wheels & $\mathrm{r}_{1}$ & 11 \\
The driving wheel radius & $\mathrm{z}$ & 200 \\
Number of drive wheel teeth & $\mathrm{r}_{2}$ & 180 \\
The guide wheel radius & $\mathrm{r}_{3}$ & 200 \\
The supporting wheel radius & $\mathrm{r}_{4}$ & \\
The tensioning wheel radius & & \\
\hline
\end{tabular}

\subsection{Design of the Wheeled Travel Mechanism}

The wheel is the most common travel mechanism used in vehicles, and tire, rim and some other components are included in the wheeled travel mechanism. The selection of the wheel was based on available data and standard parameters; rim and tire models were determined according to the design requirements of the wheel-track composite chassis and tire specifications, with selected models of 8.3-24. The rim width is $210 \mathrm{~mm}$, rim diameter is $61 \mathrm{~mm}$ (24 inches), inner rim diameter is $15 \mathrm{~mm}$ (5.9 inches), tire section width is $21 \mathrm{~mm}$ (8.3 inches), height to width ratio is 0.65 and tire diameter is $995 \mathrm{~mm}$.

\section{Theoretical Analysis of Passability}

The passability of a vehicle indicates its ability to pass through various complex roads, roadless areas and various obstacles at a high enough average speed. It depends mainly on the physical properties of the ground and the structural and geometric parameters of the vehicle. In this section, passability, including climbing performance and obstacle crossing performance, is analyzed theoretically.

\subsection{Theoretical Analysis of Climbing Performance}

The wheel-track composite chassis has an extreme slope angle during climbing, and when the slope angle exceeds the limit value, the chassis will overturn. The forces on its longitudinal driving along a slope are shown in Figure 3.

When the chassis rises at uniform speed, the torque balance equation of support point $B$ is:

$$
b G \cos \alpha-h G \sin \alpha-N l=0
$$

In the equation, $G$ is gravity; $N$ is the support force; $l$ is the distance between the support force and the support point $B ; b$ is the distance between the center of mass to the support point $B$ along the slope direction; $a$ is the distance between the center of mass to the support point $A$ along the slope direction; $\alpha$ is the slope angle; $h$ is the vertical distance between the center of mass to the slope. 


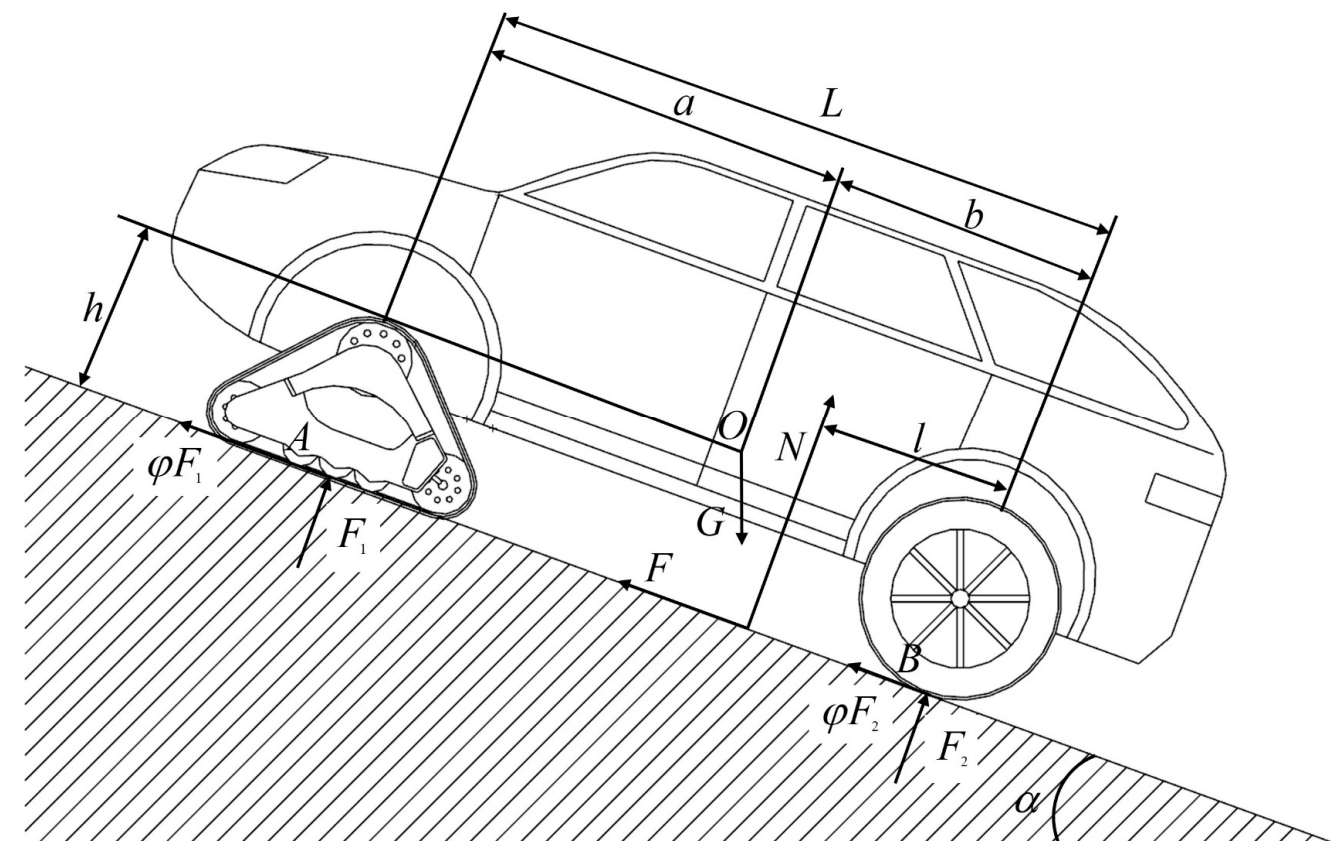

Figure 3. Schematic of longitudinal uphill running.

In order to keep the wheel-track compound chassis from tipping over when going uphill, it needs to ensure $l \geq 0$-namely, meet $b \cos \alpha-a \sin \alpha \geq 0$. It is deduced that the relationship between the working slope angle and the position of the center of mass during the longitudinal uphill movement is as follows:

$$
\alpha_{s} \leq \arctan \frac{b}{h}
$$

Replacing the relevant parameters into Equation (2), the maximum slope angle without tipping is 32.6 degrees when the chassis is driving uphill at a uniform speed along a longitudinal slope.

\subsection{Theoretical Analysis of Obstacle Crossing Performance}

In this paper, two types of obstacles, respectively vertical obstacles and trenches, are mainly considered.

\subsubsection{Theoretical Analysis of the Chassis over the Vertical Obstacle}

As is shown in Figure 4, the process can be divided into two stages. In the first stage of Figure $4 \mathrm{a}$, the track plates on the guide wheel contact the edge of the vertical obstacle and rise to the highest point; the main center of mass is in the critical state of crossing the vertical obstacle. In the second stage of Figure $4 \mathrm{~b}$, the main center of mass is over the edge of the vertical obstacle, and then the track bottom is also on the vertical obstacle and in the horizontal state. Finally, the chassis completely crosses the vertical obstacle [21].

A force analysis of the triangular tracked wheel when crossing the vertical obstacle is shown in Figure 5.

When the chassis crosses the vertical obstacle, the static balance equation is as follows:

$$
\left\{\begin{array}{l}
F_{11} \cos \alpha-f F_{11} \sin \alpha-\varphi F_{12}-\varphi F_{2}=0 \\
f F_{11} \cos \alpha+F_{11} \sin \alpha+F_{12}+F_{2}-G=0 \\
-f F_{11} \frac{e}{2}+F_{2} l-G a-\left(\varphi F_{2}+\varphi F_{12}\right) \frac{\sqrt{2} e}{2} \sin \delta-F_{12} \frac{e}{2}=0 \\
F_{2}\left(l-\frac{\sqrt{2} e}{2} \sin \delta\right)-G\left(a-\frac{\sqrt{2} e}{2} \sin \delta\right)=0
\end{array}\right.
$$




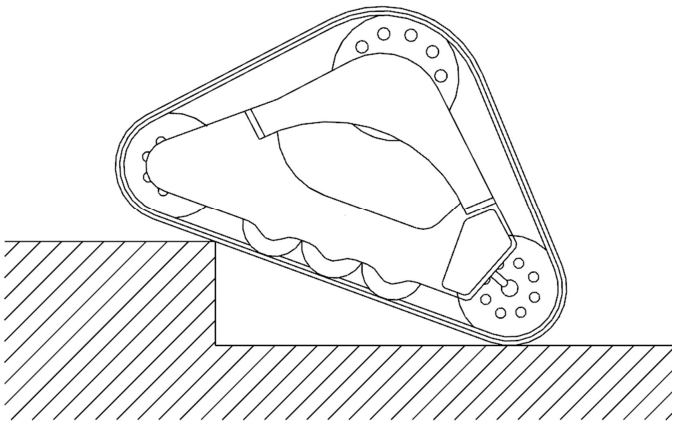

(a)

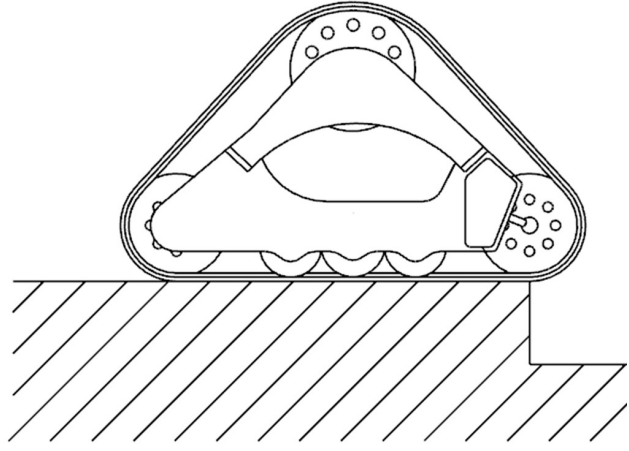

(b)

Figure 4. Two stages of the triangular tracked wheel crossing the obstacle. (a) The first stage; (b) The second stage.

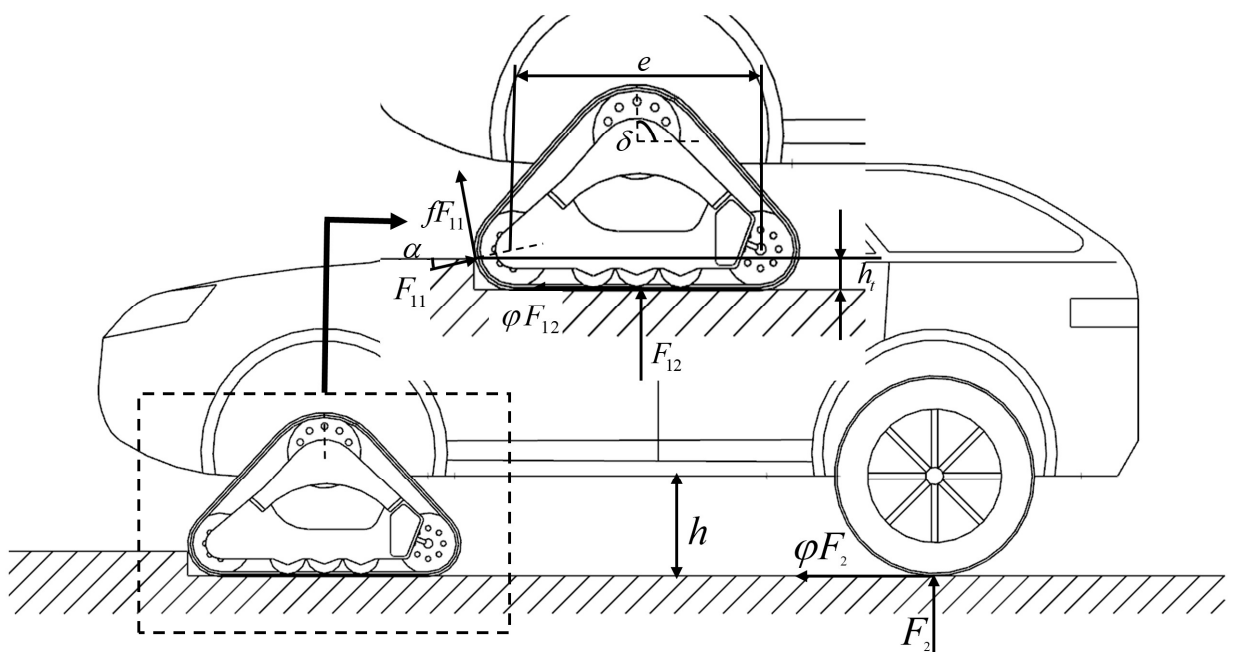

Figure 5. Analysis of triangular tracked wheel crossing obstacle.

In the equation, $G$ is the total gravity of the whole machine; $F_{11}$ is the reverse force of the vertical obstacle; $F_{12}$ is the reverse force of the ground for the triangular tracked wheel; $F_{2}$ is the reverse force of the ground for the wheeled travel mechanism; $l$ is the wheelbase; $e$ is the maximum grounding length of the track when crossing the vertical obstacle; $a$ is the distance between the center of mass and the front axis; $b$ is the distance between the center of mass and the rear axis; $h_{t}$ is the vertical obstacle height; $h$ is the distance between the center of mass and axis of the driving wheel; $\varphi$ is the attachment coefficient; $\alpha$ is the angle between $F_{11}$ and horizontal line; $\delta$ is the swing angle of the triangular tracked wheel when crossing the vertical obstacle.

The obstacle height of the triangular track climbing over the vertical obstacle still needs to meet

$$
h_{t} \leq L \sin \delta+\frac{l_{1}}{\tan \alpha}
$$

In the equation, $L$ is the grounding length of the triangular tracked wheel; $l_{1}$ is the horizontal distance between the front edge of the triangular tracked wheel contacting ground and the front edge of the guide wheel.

When replacing the relevant parameters into Equation (4) and calculating the maximum height of the vertical obstacle, it is $228 \mathrm{~mm}$.

A force analysis of the rear wheel travel mechanism when crossing the vertical obstacle is shown in Figure 6. 


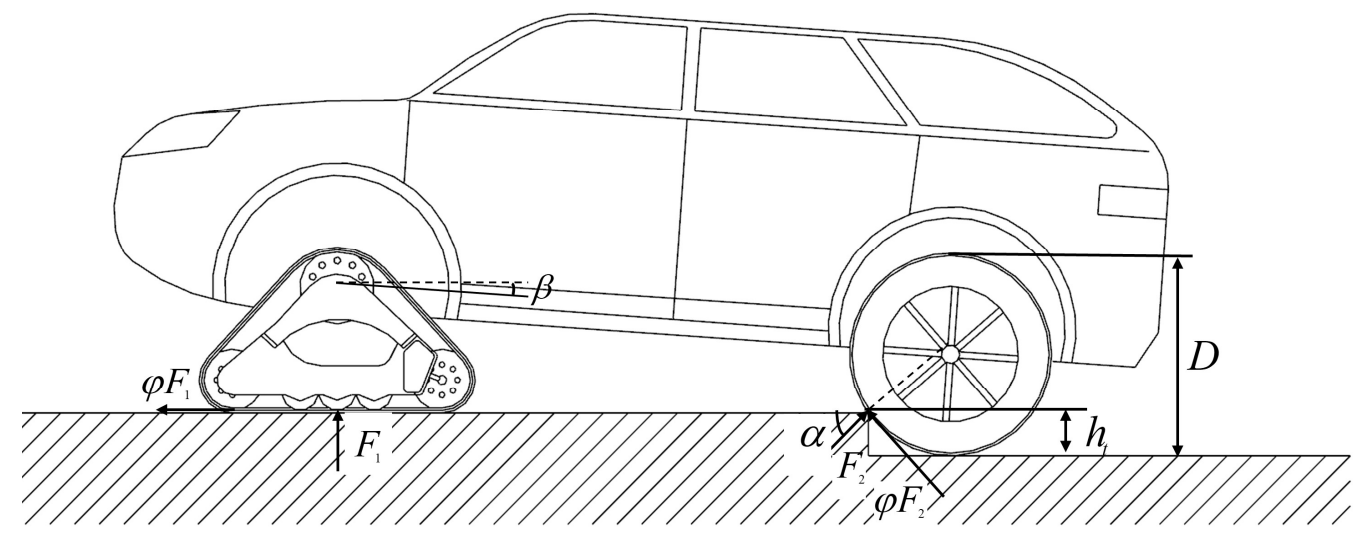

Figure 6. Analysis of wheel walking mechanism.

When the rear wheel climbs over the vertical obstacle, the static balance equation is as follows:

$$
\left\{\begin{array}{l}
\varphi F_{1}+F_{2} \sin \alpha-F_{2} \cos \alpha=0 \\
\varphi F_{2} \cos \alpha+F_{2} \sin \alpha+F_{1}-G=0 \\
\varphi F_{1}\left(0.5 D-h_{t}\right)+F_{1} l \cos \beta-0.5 \varphi F_{2} D+G b \cos \beta=0
\end{array}\right.
$$

In the equation, $F_{1}$ is the reverse force of vertical obstacle height acting on the front wheel; $F_{2}$ is load of the rear axle; $l$ is the wheelbase; $D$ is the tire diameter; $a$ is the distance between the center of mass and the front axle; $\varphi$ is the attachment coefficient; $\alpha$ is the angle between $F_{1}$ and the horizontal line.

According to the geometric relationship,

$$
\begin{gathered}
\sin \alpha=1-\frac{2 h_{t}}{D} \\
\sin \beta=\frac{h_{t}}{l}
\end{gathered}
$$

Put the upper type into Equation (7), and when the rear wheel contacts the vertical obstacle, the equation for the vertical obstacle height of the unit wheel diameter is as follows:

$$
\begin{aligned}
& \left(\sqrt{1-\left(\frac{h_{t}}{l}\right)^{2}}-\frac{\varphi h_{t}}{l}+\frac{\varphi D}{2 l}\right)\left(1-\frac{2 h_{t}}{l}\right)-\frac{\varphi D}{2 l}- \\
& {\left[\left(\frac{1+\varphi^{2}}{\varphi} \frac{a}{l}-\varphi\right) \sqrt{1-\left(\frac{h_{t}}{l}\right)^{2}}+\left(\frac{1+\varphi^{2}}{\varphi} \frac{h}{l}+\frac{D}{2 l}\right)\right] \cdot \sqrt{\frac{4 h_{t}}{D}-\left(\frac{2 h_{t}}{D}\right)^{2}}=0}
\end{aligned}
$$

According to Equation (8), the height of the vertical obstacle of the unit wheel diameter of the rear wheel, $\left(h_{t} / D\right)$, increases with decreases in $(a / l)$. In addition, the value of the rear wheel to cross the vertical obstacle is always improved when the value of $(l / D)$ is relatively large, regardless of how the quality of the whole machine is distributed between the axes.

Replacing the relevant parameters into Equation (8), the maximum height is $398 \mathrm{~mm}$.

\subsubsection{Theoretical Analysis of the Chassis over a Trench}

As shown in Figure 7, the process can be divided into three stages. In the first stage of Figure 7a, the grounded front edge of the triangular tracked wheel contacts the edge of the trench and gradually leaves the ground in a suspended position. In the second stage of Figure $7 \mathrm{~b}$, the center of mass of the triangular tracked wheel crosses the edge of the trench and is about to be suspended, with the triangular track in an inclined position. In the third stage of Figure 7c, the grounded front edge of the triangular tracked wheel reaches the other edge of the trench, and then the triangular track is horizontal and completely across the trench [22]. The width of a triangular tracked chassis across a trench is related to the 
track grounding length of the chassis and the position of the center of gravity of the entire chassis [23], and the ability of a wheel-tracked composite chassis to cross a trench in this study was evaluated using a horizontal trench, taking into account safety aspects.

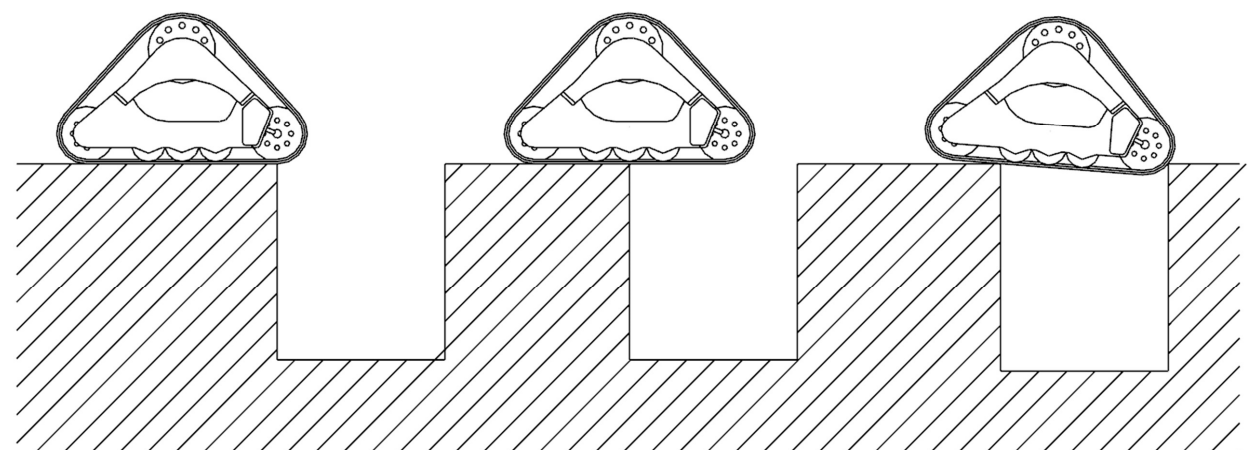

(a)

(b)

(c)

Figure 7. Three stages of the triangular tracked wheel crossing the trench. (a) The first stage; (b) The second stage; (c) The third stage.

As is shown in Figure 8, the width when the grounded front edge of the guide wheel contacts the other edge of the trench is the maximum one.

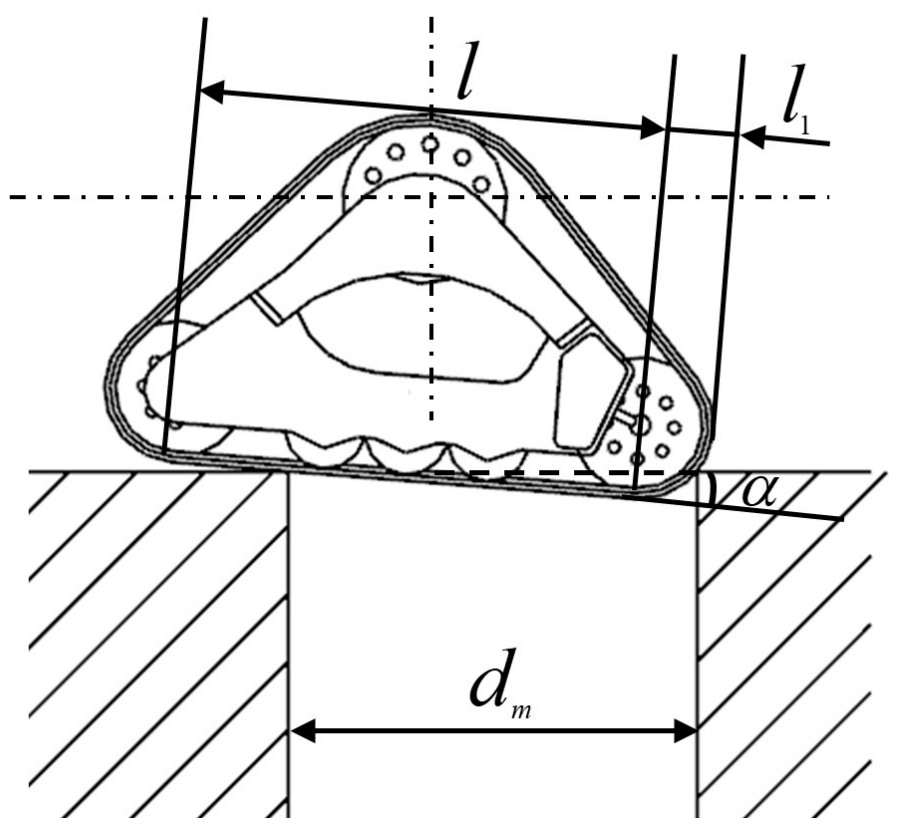

Figure 8. Force analysis of triangular tracked wheels across trenches.

The equation that the triangular tracked wheel can span the maximum width of the trench is as follows:

$$
d_{m}=l \cos \delta+\frac{l_{1} \cos (\alpha-\delta)}{\cos \alpha}
$$

Replacing the relevant parameters into the equation, the maximum width of the trench is $659 \mathrm{~mm}$.

The rear wheel travel mechanism of the composite chassis is shown in Figure 9. 


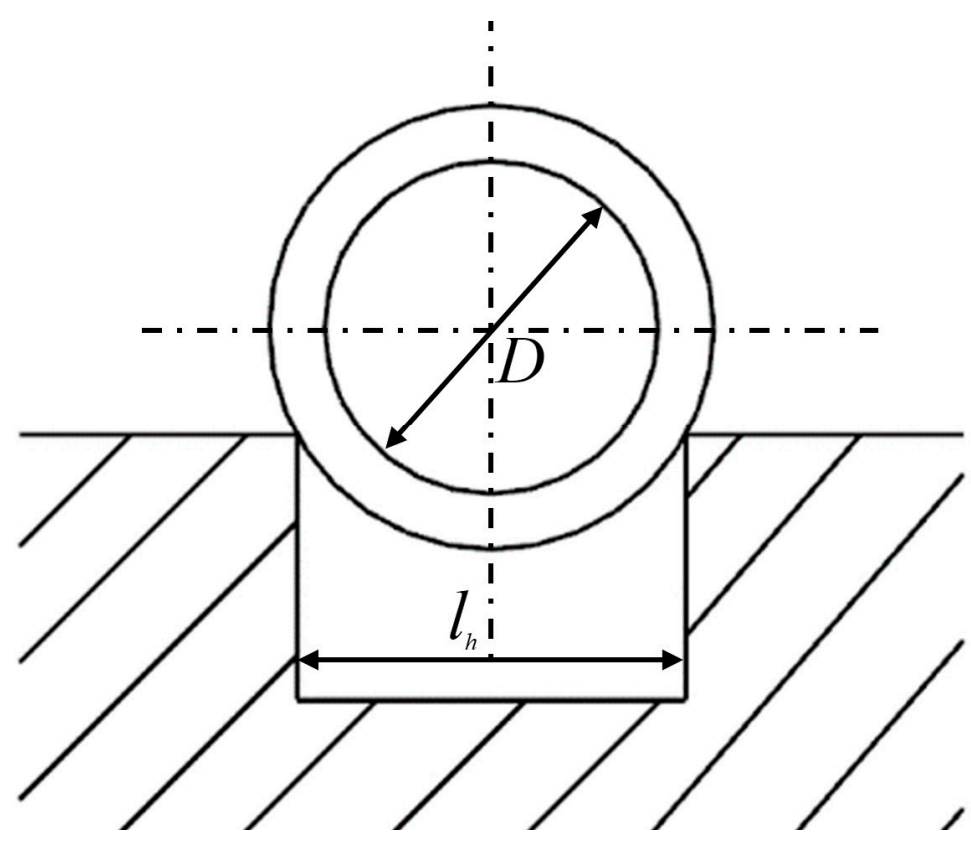

Figure 9. Schematic diagram of the wheel travel mechanism crossing the trench.

The width of the chassis across the trench and the vertical obstacle height of the unit wheel diameter are as follows:

$$
\frac{l_{h}}{D}=2 \sqrt{\left(\frac{h_{t}}{D}\right)-\left(\frac{h_{t}}{D}\right)^{2}}
$$

In the equation, $l_{h}$ is trench width; $D$ is wheel diameter.

Replacing the relevant parameters into the equation, the maximum width is $833 \mathrm{~mm}$.

\section{Dynamics Simulation Analysis Based on the RecurDyn}

RecurDyn based on multibody dynamics calculations is capable of building multidisciplinary analytical problems into an achievable coupled computational simulation platform.

\subsection{Dynamics Theory of Multibody Systems}

Multibody dynamics equations of a system are developed for the analysis of the multibody dynamics of a structural system [24]. The structure to be analyzed is geometrically modeled, and then realistic motion constraints and motion conditions are added to the structure. Finally, the structural system is analyzed using the relevant theory and dynamics equations in multibody dynamics analysis software to obtain simulation results.

\subsubsection{Dynamics Analysis of Multibody Systems}

The analysis method based on "computational multibody system dynamics" refers to the preparation of the corresponding program based on the theory of dynamics analysis, using the high-speed solution ability of a computer so as to achieve the purpose of the analysis. The main process can be divided into two parts: modeling and solution [25].

\subsubsection{Principles of Automatic Modeling}

The kinetic equations described for a multibody system using absolute coordinates are generally in the form of

$$
\left\{\begin{array}{l}
A \ddot{q}+\Phi_{q} \lambda=B \\
\Phi(q, t)=0
\end{array}\right.
$$


In the equation, $q$ is a position coordinate array; $q \in R^{n}$ is the acceleration vector; $\lambda \in R^{m}$ is the Lagrange multiplier; $t \in R$ is time; $A \in R^{n \times n}$ is the inertia matrix of system; $B$ is the system constant; $\Phi_{q} \in R^{m \times n}$ is the Jacobian constraint matrix; $\Phi \in R^{m}$ is the constraint equation.

\subsubsection{Establishment of the Control Equation}

The control equations are first established by separating each rigid body independently, then establishing the dynamic equations of each rigid body based on Lagrange's equations, and then listing the constraint equations through the connection relationship between the kinematic suits of the components. For a single rigid body, its Lagrange's equation is

$$
\frac{d}{d t}\left(\frac{\partial L}{\partial \dot{q}_{k}}\right)-\frac{\partial L}{\partial q_{k}}=Q_{k}, k=1,2, \cdots, n
$$

In the equation, $q_{k}$ is the generalized coordinate of the $k$ th rigid body in the system; $L$ is the Lagrangian term, $L=T-W$ is defined and $T$ and $W$ represent the system kinetic energy and potential energy, respectively; $Q_{k}$ is the generalized force of the $k$ th rigid body; $n$ is the number of rigid bodies.

The constraint equation for the system is

$$
\Psi_{i}(q, t)=0, i=1,2, \cdots, m
$$

In the equation, $m$ is the number of constraints; the formula for Equation (13) in matrix form is

$$
\begin{gathered}
\frac{d}{d t}\left(\frac{\partial T}{\partial \dot{q}}\right)-\frac{\partial T}{\partial q}=Q_{F}-K q \\
T=\frac{1}{2} \dot{q}^{T} M \dot{q}
\end{gathered}
$$

In the formula, $Q_{F}$ is all the active force sustained by the rigid body, removing the generalized force corresponding to the generalized elastic force part; $K$ is the stiffness matrix; $M$ is the system mass matrix. Bring formula for Equation (15) into the formula for Equation (14), we get

$$
M \ddot{q}+q=Q_{F}+Q_{V}
$$

Among

$$
Q_{V}=-\dot{M} \dot{q}+\frac{\partial}{\partial q}\left(\frac{1}{2} \dot{q}^{T} M \dot{q}\right)
$$

is a generalized force of the corresponding velocity quadratic term. Change the formula for Equation (17) into the following matrix block form:

$$
\begin{aligned}
& {\left[\begin{array}{lll}
m_{R R} & m_{R \theta} & m_{R f} \\
m_{R \theta}^{T} & m_{\theta \theta} & m_{\theta f} \\
m_{R f}^{T} & m_{\theta f}^{T} & m_{f f}
\end{array}\right]\left[\begin{array}{c}
\ddot{R}_{0} \\
\dot{\omega} \\
q_{f}
\end{array}\right]+\left[\begin{array}{lll}
0 & 0 & 0 \\
0 & 0 & K_{f f}
\end{array}\right]\left[\begin{array}{c}
R_{0} \\
\theta \\
q_{f}
\end{array}\right]} \\
& =\left[\begin{array}{l}
Q_{R} \\
Q_{\theta} \\
Q_{f}
\end{array}\right]+\left[\begin{array}{l}
Q_{V R} \\
Q_{V \theta} \\
Q_{V f}
\end{array}\right]
\end{aligned}
$$

$\ddot{R}_{0}, \dot{\omega}$ in the obtained formula for Equation (18) is the system flexible body dynamic equation. $R_{0}$ is the amount of movement of the rigid body; $\theta$ is rotation of the rigid body; the superscript $T$ is a transposition; $\ddot{R}_{0}$ is movement acceleration; $\dot{\omega}$ is rotational acceleration; $q_{f}$ is deformation.

After combining the various rigid bodies of the system, the Lagrange equation is

$$
M q+K q+C_{q}^{T} \lambda=Q_{F}+Q_{V}
$$


and the constraint equation is

$$
\Psi(q, t)=0
$$

The combination of the formulas for Equations (19) and (20) is the flexible multibody systems control equation. $C_{q}$ is the Jacobian matrix of the constraint equation [26].

\subsection{Establishment of the 3-Dimensional Model}

Based on multibody dynamics, RecurDyn provides a specialized toolkit that can be more convenient for specific applications in relevant industries, such as its track creation toolbox, which can conduct motion simulation of various track driving mechanisms [27].

As shown in Figure 10, this study used UG software to synthesize the frame and tires into a rigid body so that the entire mobile ground is divided into two parts: the tracked travel mechanism and the wheeled travel mechanism-frame The structural parameters of the wheel composite chassis are shown in Table 2.

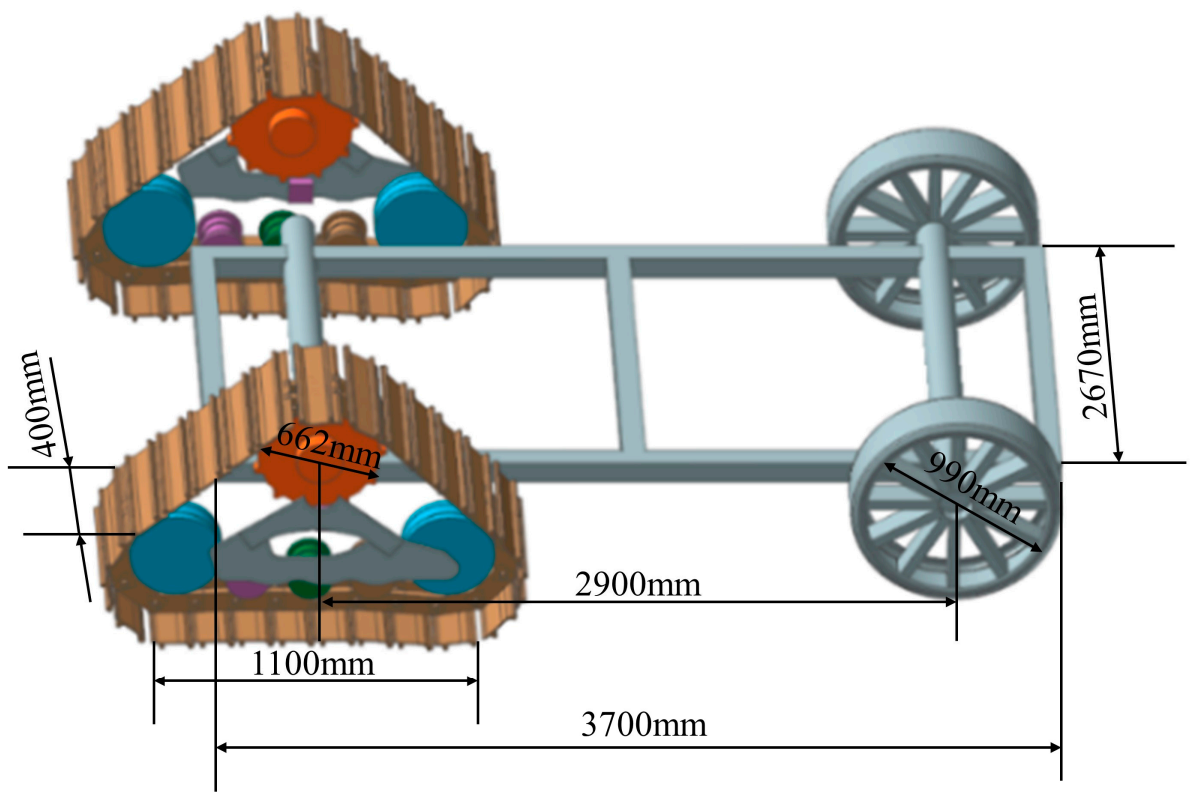

Figure 10. Overall model of the wheel-track composite chassis.

Table 2. Structural parameters of the wheel-track composite chassis.

\begin{tabular}{ccc}
\hline Structural Parameters of Wheel Composite Chassis & Unit & Value \\
\hline Whole-vehicle quality & $\mathrm{kg}$ & 7000 \\
Chassis size & $\mathrm{mm} \times \mathrm{mm} \times \mathrm{mm}$ & $3700 \times 2670 \times 2450$ \\
Tire radius & $\mathrm{mm}$ & 495 \\
Driving wheel diameter & $\mathrm{mm}$ & 662 \\
Wheelbase & $\mathrm{mm}$ & 2900 \\
Track ground length & $\mathrm{mm}$ & 1100 \\
Track width & $\mathrm{mm}$ & 400 \\
\hline
\end{tabular}

\subsection{Constraints of the Model}

To effectively simulate the driving process of the wheel composite chassis, constraints are necessary to be added between the components, as shown in Table 3. 
Table 3. Constraints between the components of the wheel-track composite chassis.

\begin{tabular}{ccc}
\hline Constraint Form & Matrix & Movement Body \\
\hline Fixed deputy & Frame & Track support frame \\
Fixed deputy & Frame & Tire \\
Rotary side & Frame & Driving wheel \\
Rotary side & Track support frame & Supporting wheel \\
Rotary side & Track support frame & Guide wheel \\
Rotary side & Track support frame & Tensioning wheel \\
Contact force & Ground & Tire \\
\hline
\end{tabular}

The fixed subsets are added between the track support frame and the frame, and the same restraint is added between the tire and the frame. The rotating subsets are added between the drive wheels and the frame, and the same restraint is added between the supporting wheels, tensioning wheels, guide wheels and the track support frame. Finally, the solid contact between the wheels and the ground is established.

\subsection{Simulation Analysis of Wheel-Track Composite Chassis Dynamics}

In this section, based on RecurDyn, the wheel-track composite chassis is simulated under different working conditions to analyze its performance parameters.

\subsubsection{Dynamics Simulations in Different Terrains}

According to the structural parameters of a rescue vehicle driving system, the force of a triangular crawler was analyzed by $\mathrm{Hu}$ and some others [28], and the corresponding multibody dynamics model was established by using the Track-HM module in the multibody dynamics software RecurDyn. By changing the vehicle speed, pre-tensioning force and road surface, which affect the crawler tension force, multiple sets of simulation calculations were performed to obtain the force of the crawler during the motion cycle under different working conditions.

The software of RecurDyn can build ground models of hard and soft pavement types by setting different parameters [29]. The simulation shows a track tension diagram and a drive wheel torque diagram traveling in both terrains, as shown in Figure 11.

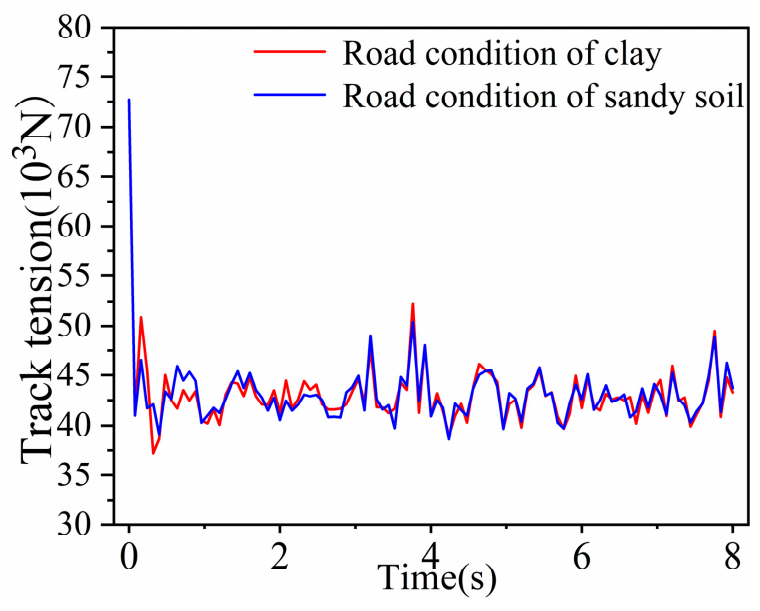

(a)

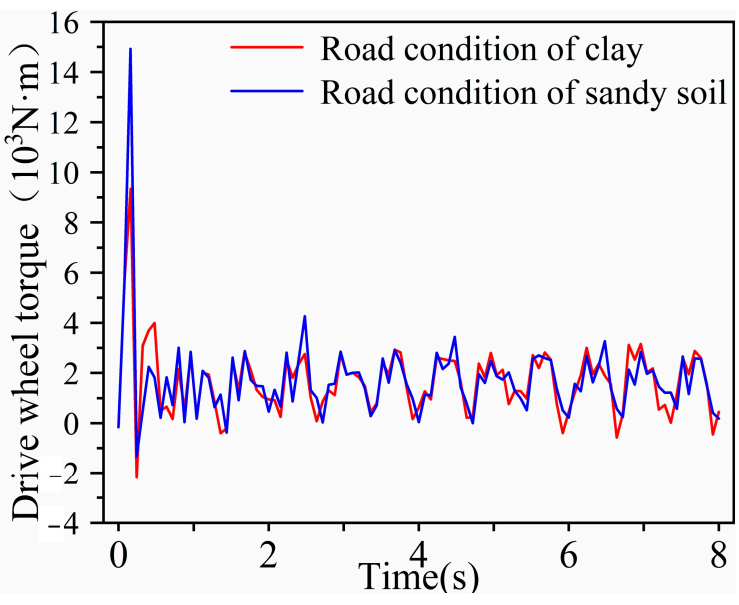

(b)

Figure 11. The simulation curves of a wheel-track composite chassis in different terrains: (a) tensiontime curve; (b) torque-time curve.

The blue curve in the figure represents the wheel-track composite chassis on clay, and the green curve represents the chassis on sandy loam. According to Figure 11, the simulation results show that the chassis can both pass through clay and sandy loam terrain and that the track tension and driving torque are similar. According to Figure 11a, the 
track tension value fluctuates above and below $43 \mathrm{kN}$, reaching a minimum at $0.3 \mathrm{~s}$ and a maximum at $3.8 \mathrm{~s}$. According to Figure $11 \mathrm{~b}$, the driving torque fluctuates above and below $23 \mathrm{kN}$, reaching a minimum driving torque at $0.1 \mathrm{~s}$ and a maximum at $0.2 \mathrm{~s}$. After $1.4 \mathrm{~s}$, the driving torque fluctuated more stably as the chassis traveled on clay.

\subsubsection{Dynamics Simulation of Different Slope Angles}

Chen and some others [21] studied a small hedge trimmer crawler chassis, and they analyzed the lateral and longitudinal stability of the crawler chassis on slopes and the overrunning performance in the process of going over vertical walls and crossing trenches in a similar way. The theoretical value of the maximum climbing of the wheel-track composite chassis was $36.5^{\circ}$, while for most agricultural land, the maximum slope value is not more than $25^{\circ}$, so the slope values selected for the simulation were $25^{\circ}$ and $36.5^{\circ}$. The same parameters derived from the wheel-track composite chassis under two kinds of slope driving were imported into a graph for comparison and analysis, so as to derive the change law of each parameter during the climbing process of the chassis, as shown in Figure 12.

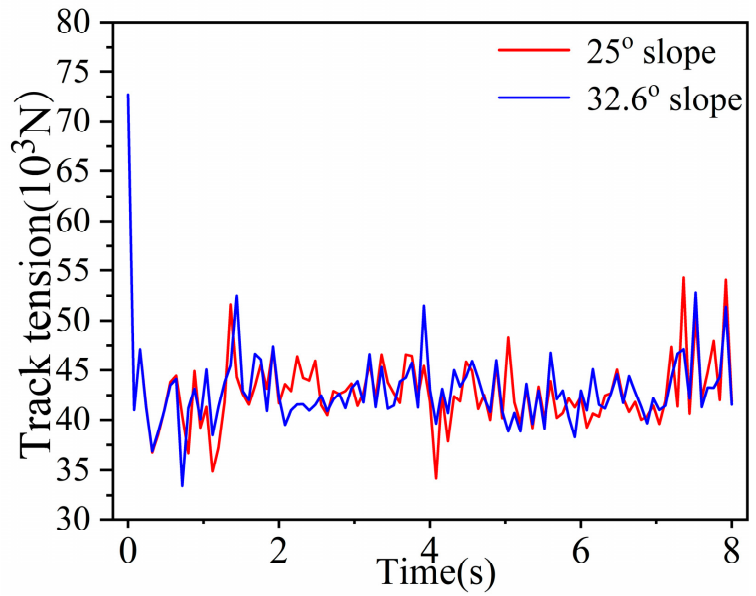

(a)

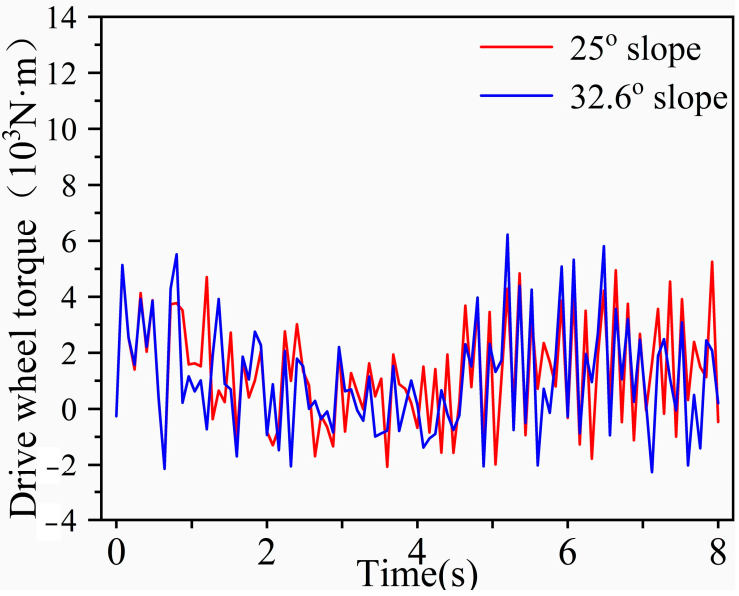

(b)

Figure 12. The simulation curves of a wheel-track composite chassis with different climbing: (a) tension-time curve; (b) torque-time curve.

The blue curve in the figure represents the chassis on a $25^{\circ}$ slope, and the green curve represents the chassis on a $32.6^{\circ}$ slope. According to Figure 12, the simulation results show that a slope of $32.6^{\circ}$ can be passed. When the chassis travels on the $25^{\circ}$ slope, the track tension fluctuates at $42 \mathrm{kN}$, reaching the minimum at $4.1 \mathrm{~s}$ and the maximum at $7.3 \mathrm{~s}$. As the chassis travels on a $32.6^{\circ}$ ramp, according to Figure $12 \mathrm{a}$, the track tension fluctuates around $44 \mathrm{kN}$, reaching a minimum at $0.7 \mathrm{~s}$ and a maximum at $7.5 \mathrm{~s}$. According to Figure $12 \mathrm{a}$, the driving torque fluctuation of the chassis when driving on the $32.6^{\circ}$ slope is greater, and the maximum driving torque is greater than the maximum value when the chassis is driving on the $25^{\circ}$ slope, which shows that when the chassis is driving on the larger slope, the required driving force will be greater.

\subsubsection{Dynamics Simulation of Different Vertical Obstacle Heights}

The maximum vertical obstacle height that the wheel-track composite chassis can pass is $228 \mathrm{~mm}$, so the vertical obstacle height was $200 \mathrm{~mm}$ and $228 \mathrm{~mm}$ during the simulation analysis, as is shown in the Figure 13. 


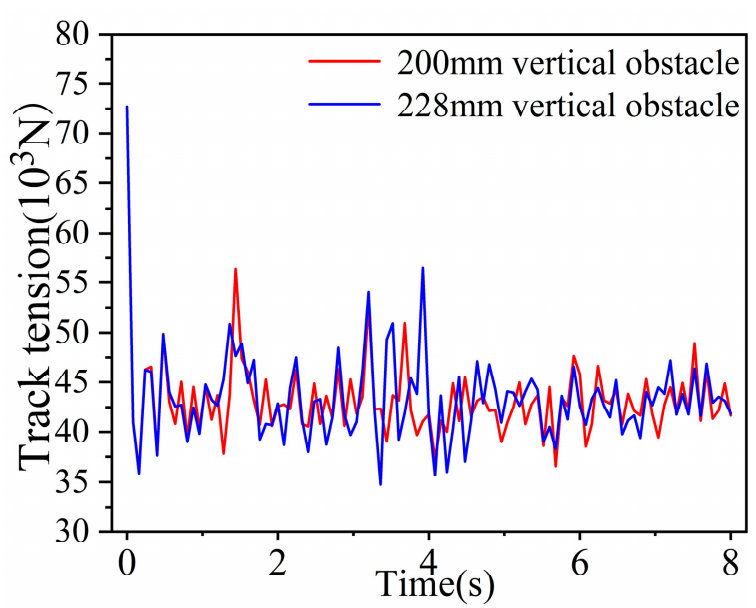

(a)

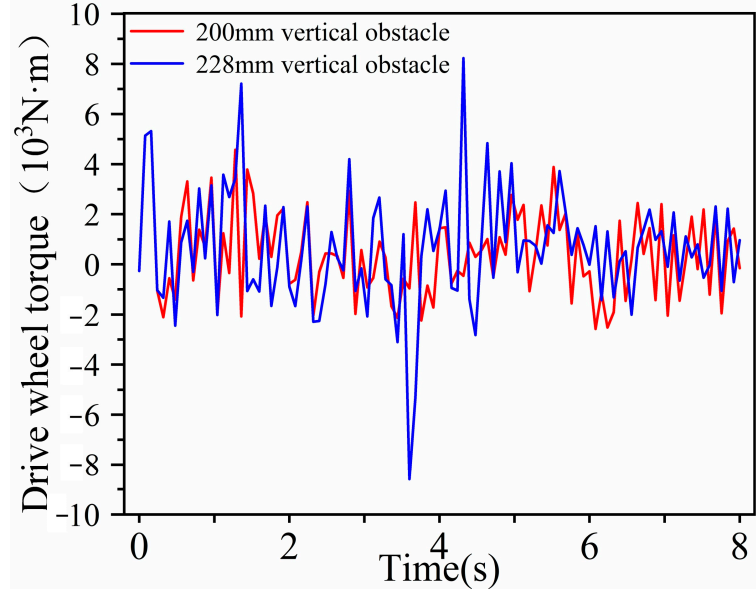

(b)

Figure 13. The simulation curves of a wheel-track composite chassis with different vertical obstacle heights: (a) tension-time curve; (b) torque-time curve.

The blue curve in the figure represents a height of vertical obstacle of $200 \mathrm{~mm}$, and the green curve represents a height of $228 \mathrm{~mm}$. According to Figure 13, the simulation results show that the composite chassis can cross a vertical obstacle with a height of $228 \mathrm{~mm}$. When the chassis crosses a $200 \mathrm{~mm}$ vertical obstacle, as shown in Figure 13a, the track tension fluctuates around $43 \mathrm{kN}$, reaching the minimum at $1.1 \mathrm{~s}$ and the maximum at $4.2 \mathrm{~s}$. As the chassis crosses a $228 \mathrm{~mm}$ vertical obstacle, the track tension fluctuates around $45 \mathrm{kN}$, reaching a minimum at $3.3 \mathrm{~s}$ and a maximum at $3.9 \mathrm{~s}$. The chassis fluctuates when crossing a $228 \mathrm{~mm}$ vertical obstacle, and the required driving torque is greater than the chassis when crossing a $200 \mathrm{~mm}$ vertical obstacle.

\subsubsection{Dynamics Simulation of Different Trench Widths}

The maximum trench width of the wheel-track composite chassis is $659 \mathrm{~mm}$, and the two sets of parameters were $600 \mathrm{~mm}$ and $659 \mathrm{~mm}$ during the simulation analysis, as shown in the Figure 14.

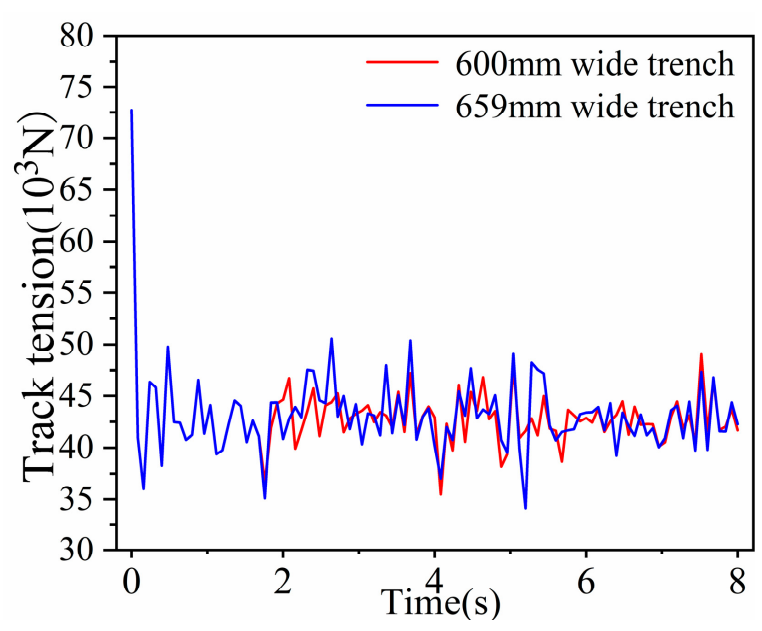

(a)

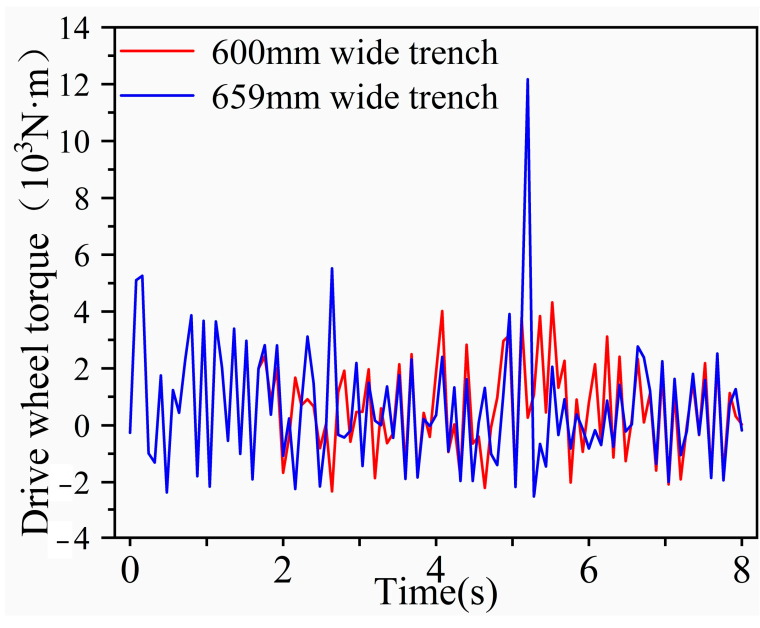

(b)

Figure 14. The simulation curves of a wheel-track composite chassis with different trench widths: (a) tension-time curve; (b) torque-time curve.

The blue curve in the figure represents a trench width of $600 \mathrm{~mm}$ and the green curve represents a trench width of $659 \mathrm{~mm}$. According to Figure 14, the simulation results show that a trench width of $659 \mathrm{~mm}$ can be passed through. The chassis fluctuates around $40 \mathrm{kN}$ 
across the width of $600 \mathrm{~mm}$ trenches, reaching a minimum at $3.6 \mathrm{~s}$ and a maximum at $5.2 \mathrm{~s}$. According to Figure $14 \mathrm{a}$, the track tension of the track fluctuated at $42 \mathrm{kN}$ across a trench width of $659 \mathrm{~mm}$, reaching a minimum at $3.5 \mathrm{~s}$ and a maximum at $4.3 \mathrm{~s}$. According to Figure $14 \mathrm{~b}$, the driving torque required for the chassis across a $659 \mathrm{~mm}$ trench is greater than that required across a $600 \mathrm{~mm}$ trench.

\section{Conclusions}

A wheel-track composite chassis was designed to improve the passability of a vehicle. Combined with the model of the chassis built by RecurDyn, a simulation analysis was carried out to obtain the change in the track tension and driving torque.

(1) Through the passing theoretical analysis of the wheel-track composite chassis and the chassis model simulation analysis, the chassis can pass through clay and sandy loam terrain, climb over a slope of $36.2^{\circ}$, and can cross the height of a vertical obstacle of $228 \mathrm{~mm}$ and the width of a trench of $659 \mathrm{~mm}$.

(2) The track tension fluctuated around a certain value regardless of the road surface on which the wheel-track composite chassis was driven. When the chassis was driving on a flat road, the fluctuation of the track tension value was the smallest. The fluctuation of the track tension value was greater when the chassis was going uphill and through obstacles.

(3) High driving torque was required when the tracked wheels passed over inclined, obstructed and trenched surfaces. The chassis was designed to improve the vehicle's ability to operate in complex and diverse unstructured conditions. Therefore, we should focus on the design and study of the drive wheels.

Author Contributions: Y.L. performed the data analyses and wrote the manuscript; L.Z. contributed to the conception of the study; T.S. performed the data curation; T.L. performed the data presentation; F.L. contributed significantly to analysis and manuscript preparation. All authors have read and agreed to the published version of the manuscript.

Funding: This research was partly supported by the National Natural Science Foundation of China under grant 51605215 and partly by the China Postdoctoral Science Foundation under grant 2019T120450, the Qing Lan Project (Teacher Su 2019 [3]).

Conflicts of Interest: The authors declare no conflict of interest.

\section{References}

1. Qu, J.; Zhong, W.B. Design and obstacle-surmounting performance analysis of wheel-track transformable wheel. J. South China Univ. Technol. (Nat. Sci. Ed.) 2013, 41, 119-124.

2. Lin, J.; Goldenberg, A.A. Development of a Terrain Adaptive Tracked Vehicle and Its Derivative-Dual Mode Vehicle. In Proceedings of the 1st WRC Symposium on Advanced Robotics and Automation, Beijing, China, 16 August 2018 ; pp. $302-307$.

3. Liu, Y.; Xie, X.; Zhou, X.; Wang, L. Summary of research on wheel-track composite chassis. Mod. Manuf. Technol. Equip. 2019, 4, 87-90.

4. Luo, Z.R.; Shang, J.Z.; Wei, G.W.; Ren, L. A reconfigurable hybrid wheel-track mobile robot based on Watt II six-bar linkage. Mech Mach. Theory 2018, 128, 16-32. [CrossRef]

5. Wang, S.K.; Meng, X.D.; Shang, H.P. Design of wheel-tracked stair-climbing wheelchair. J. Mech. Transm. 2013, 37, 156-159.

6. Li, C.R.; Shi, C.H.; Huang, L.; Li, N. Dynamic simulation analysis of a novel wheel-tracked mobile chassis. J. Mach. Des. 2016, 33, 95-99.

7. Bian, H.R.; Zi, X.Y.; Wang, H.T.; Tang, Y.Q.; Zeng, F.Q. Design and experiment of a rescue-search robot based on deformable track. J. Ordnance Equip. Eng. 2017, 38, 143-146.

8. Janez, P.; Jure, R.; Sebastjan, S.; Marko, M.; Matjaz, M. All-Terrain Wheelchair: Increasing Personal Mobility with a Powered Wheel-Track Hybrid Wheelchair. IEEE Robot. Autom. Mag. 2017, 24, 26-36.

9. Editorial Department of China Journal of Highway and Transport. Review on China's automotive engineering research progress: 2017. China J. Highw. Transp. 2017, 30, 1-197.

10. Kim, Y.G.; Kwak, J.H.; Kim, J.; An, J.; Lee, K.D. Adaptive Driving Mode Control of Mobile Platform with Wheel-Track Hybrid Type for Rough Terrain in the Civil Environment. In Proceedings of the International Conference on Control, Automation and Systems, Gyeonggi-do, Gyeonggi-do, Korea, 27-30 October 2010; pp. 86-90.

11. Michaud, F.; Letourneau, D.; Arsenault, M.; Bergeron, Y.; Cadrin, R.; Gagnon, F.; Legault, M.A.; Millette, M.; Paré, J.F.; Tremblay, M.C.; et al. Multi-Modal Locomotion Robotic Platform Using Leg-Track-Wheel Articulations. Auton. Robot. 2005, 18, 137-156. [CrossRef] 
12. Kim, J.H.; Lee, C.G. Variable Transformation Shapes of Single-Tracked Mechanism for A Rescue Robot. In Proceedings of the 2007 IEEE International Conference on Control, Automation and Systems, Seoul, Korea, 17-20 October 2007; pp. $1057-1061$.

13. Sun, Y.Q. Rubber-Tracked Chassis Structure Design and Experimental Simulation; Daqing Petroleum institute: Daqing, China, 2010.

14. Zhou, H.L.; Huang, X.H.; Zou, S.Y.; Liu, W.M. Research on the traveling device of track combine harvester. Mod. Agric. Equip. 2006, 5, 47-49.

15. Lv, K.; Mu, X.H.; Du, F.P.; Guo, H.L.; Zhao, X.D. Review of key design problems of heavy-duty conversion rubber track system. J. Acad. Armored Force Eng. 2016, 30, 29-38.

16. Lv, K.; Mu, X.H.; Guo, H.L.; Xue, W.B.; Wang, Z.Y.; Xu, L. Modeling and testing on track perimeter of conversion rubber track assembly. Trans. Chin. Soc. Agric. Mach. 2016, 47, 329-340.

17. Ji, Y.C.; Wang, Q.K. Classification and application characteristics of bulldozers' wheel. J. Chin. Agric. Mech. 2018, $39,22-25$.

18. Li, N.; Zhou, J.; Zhang, H.; Cui, Z.K.; Jiao, Z.Y.; Jiang, W. Development status and analysis of triangular rubber track wheel technology. J. Chin. Agric. Mech. 2019, 40, 209-219.

19. Yang, X. Virtual Design and Performance Analysis of Run-Flat Inserts; Jilin University: Jilin, China, 2007.

20. Yang, D.M. Design and Simulation Analysis of a Replaceable Triangle Track Wheel of 50 Type for Military; Harbin Institute of Technology: Harbin, China, 2016.

21. Chen, J.Q.; Huang, R.Z.; Mo, R.X.; Qiang, H.; Liu, X.; Xu, G.W. Analysis and simulation of obstacle crossing performance of tracked chassis of small hedge trimmer based on Recurdyn. J. Chin. Agric. Mech. 2020, 41, 89-98.

22. Zhang, G.H. Study on the Trafficability of Wheeled and Triangle Tracked Multifunctional Skidders. Master's Thesis, Northeast Forestry University, Harbin, China, 2020.

23. Wang, F.; Yang, L.; Xie, S.Y.; Ma, Y.; Zhang, J.; Duan, T.Y. Design and research of a triangular track orchard power chassis. J. Agric. Mech. Res. 2019, 41, 91-96.

24. Chen, H.Y.; Yuan, Y.N.; Zhang, T.; Yu, H.S. Starting process on hybrid electric vehicle with floating ISG motor. J. Jiangsu Univ. (Nat. Sci. Ed.) 2010, 31, 403-407.

25. Wu, P. Research of Modeling and Numerical Solution for Dynamics of Multibody Systems; Sichuan University: Chengdu, China, 2003.

26. Hu, J.Z.; Huang, L.; Kang, S.H.; Shi, C.H.; Li, N. Structural design and dynamical simulation analysis on wheel-tracked variant wheel. Chin. J. Constr. Mach. 2015, 13, 130-134.

27. Jiao, X.J.; Zhang, J.W.; Peng, B.B. Recurdyn Multibody Systems Optimization Simulation Technology, 3rd ed.; Tsinghua University Press: Beijing, China, 2010; pp. 57-58.

28. Hu, C.B.; Tu, Z.Q.; Yang, X.; Jiang, C.M.; Pan, M. Modeling and Simulation of Triangle Crawler Driving System Based on Recurdyn. J. Ordnance Equip. Eng. 2020, $41,7$.

29. Liu, S.; Xie, N.; Zhang, T. Design and simulation of small crawler chassis for mountain areas. J. Mach. Des. 2020, 37, 115-122. 